\title{
Razonamiento clínico en kinesiología mediante aprendizaje basado en casos
}

\section{Clinical reasoning in kinesiology by case-based learning}

Felipe Contreras-Briceño ${ }^{1 *}$; Yenny Villaseca'; Maximiliano Espinosa-Ramírez'; Gregory Villarroel'; Gonzalo Moscoso'; Magdalena Chamorro'; Natalia Nicoletti'; Macarena Espinoza'; Patricio García'; Tiziana Fernández'; Cynthia Rojas-Muñoz ${ }^{1}$

\section{Resumen}

Introducción: En cursos clínicos con gran número de estudiantes, las experiencias clínicas con pacientes reales son limitadas, dificultando el logro de objetivos de aprendizaje. La didáctica aprendizaje basado en casos (ABC) promueve el pensamiento crítico y trabajo coolaborativo, aspectos esenciales para desarrollar competencias profesionales. El objetivo de este estudio fue reportar si la incorporación de la metodología $A B C$ en una asignatura clínica curricular promueve el razonamiento clínico en la formación en kinesiología.

Metodología: En la asignatura curricular "evaluación cardiorrespiratoria en kinesiología", 10 grupos de 7 estudiantes desarrollaron casos clínicos de temas disciplinares seleccionados bajo criterio de jueces por expertos del área, y lo presentaron al resto de sus compañeros. Un académico guió la reflexión del tema tratado en el ABC, fomentando la discusión entre los estudiantes. Al finalizar la asignatura se evaluó la percepción de la didáctica educativa mediante encuesta y logro de objetivos de aprendizaje con indicadores académicos. Resultados: Los estudiantes reportaron gran satisfacción con la metodología, mayor preparación para actividades de campo clínico y mejoras en sus habilidades comunicacionales. El promedio obtenido en las interrogaciones y en las actividades clínicas fue superior a versiones previas de la asignatura, aumentando el porcentaje de aprobación y satisfacción con el curso. Conclusión: La incorporación de la didáctica de $\mathrm{ABC}$ fomentó el razonamiento clínico, reflexión y habilidades comunicacionales mejorando el rendimiento académico y promoviendo competencias profesionales. Como producto final se elaboró un libro de descarga libre con los temas tratados en los ABC, titulado: "Identificando problemas kinesiológicos: aprendizaje basado en casos".

Palabras clave: razonamiento clínico; metodología educativa; aprendizaje basado en casos; pensamiento crítico; reflexión.

\begin{abstract}
Introduction: In clinical courses with a large number of students, clinical experiences with real patients are limited, difficult to achieve the learning objectives. The 'Case-Based Learning' $(\mathrm{CBL})$, like educational methodology, promotes critical thinking and improve collaborative work, which are essential aspects of the development of professional skills. The objective of this study was to report how the incorporation of the CBL methodology in a clinical course promoted the clinical reasoning in kinesiology students. Methodology: In the curriculum subject ¿Cardiorespiratory assessment in kinesiology, ten groups of seven students analyzed different clinical cases and presented them to their classmates. The CBL topics were selected by academic experts, who also guided and encouraged the discussion among the students. An online survey at the end of the course assessed perceptions students participated in this methodology. The final academic indicators assessed the learning objectives of the course achieved by students that participated with this methodology. Results: The students reported high satisfaction with the CBL methodology. They showed better preparation for clinical experiences and an improvement in their communication skills. The final marks obtained in the interrogations and the clinical practice activities were higher than in previous years. There was an increase in academic approval and student satisfaction with the course. Conclusion: The incorporation of the CBL methodology enhanced clinical reasoning, reflection, and communication skills, improving academic performance, and promoting professional skills. The topics covered in the CBLs conformed a free download book, entitled: «ldentifying kinesiology problems: case-based learning.»
\end{abstract}

Keywords: clinical reasoning; teaching methodology; case-based learning; critical thinking; reflection.

Fecha de envío: 7 de enero de 2020 - Fecha de aceptación:12 de junio de 2020

(1) Departamento Ciencias de la Salud, Facultad de Medicina, Pontifica Universidad Católica de Chile.

*Autor correspondiente: fcontrerasb@uc.cl 


\section{Introducción}

La competencia profesional en las carreras de la salud se define como el uso continuo de un adecuado razonamiento clínico, destrezas técnicas, lenguaje, valores, y procesos de reflexión que les permite a los profesionales realizar y fundamentar adecuadamente la intervención de salud hacia las comunidades que éstas sirven (Epstein \& Hundert, 2002).

El plan de estudios de la carrera de kinesiología incorpora en tercer año las primeras asignaturas clínicas, cuyo objetivo es que los estudiantes desarrollen adecuadamente el proceso de evaluación con pacientes reales en actividades prácticas de campo clínico (visitas clínicas), identificando así problemas kinesiológicos que sustenten la resolución terapéutica a plantear en niveles superiores. Al respecto, la asignatura de evaluación cardiorrespiratoria en kinesiología (KIN304a) tiene por objetivo que los estudiantes desarrollen un adecuado razonamiento clínico a partir de la evaluación con enfoque cardiorrespiratorio que les permita ser capaces de formular un diagnóstico kinesiológico. Para ello, en versiones anteriores de la asignatura (cohortes 2014 a 2017) se han desarrollado diferentes didácticas educativas, destacando la experiencia en visitas clínicas como la más valorada por los estudiantes. Sin embargo, los escenarios clínicos que ofrecen estas actividades varían en complejidad, pudiendo afectar el desarrollo de razonamiento clínico y además, dejan escaso tiempo para la autorreflexión, aspectos que dificultan en el estudiante la regulación de su aprendizaje (Issenberg \& Scalese, 2015). Una forma de favorecerlo es aumentar el número de visitas clínicas, lo cual se ve limitado por el elevado número de estudiantes por nivel y una acotada alianza con campos clínicos, aspectos que dificultan el logro de objetivos de aprendizaje de la asignatura. Además, los estudiantes perciben dificultades en la aplicación e integración de contenidos teóricos con la evaluación producto del escaso tiempo de contacto clínico con pacientes, lo cual coincide con la opinión de tutores de campo clínico quienes destacan el sólido nivel de contenidos teóricos de los estudiantes, pero dificultad en su aplicación a la realidad clínica. Lo anterior, sostiene la necesidad de incorporar nuevas metodologías docentes que promuevan el desarrollo del razonamiento clínico, pensamiento crítico y reflexión, donde se integren contenidos teóricos aplicados a situaciones clínicas reales, con el fin de desarrollar visitas clínicas más enriquecedoras y así promover el logro de los objetivos de aprendizaje de la asignatura.

El aprendizaje basado en casos ( $A B C$ ) es una metodología educativa que promueve el desarrollo del pensamiento crítico gracias a la discusión grupal de una situación que posee elementos derivados de la realidad. Los casos clínicos que se plantean no se solucionan, ni resuelven, ni corrigen; sólo se discuten (Williams, 2005). La discusión reflexiva favorece que los estudiantes relacionen contenidos teóricos adquiridos previamente con situaciones reales, y al ser un trabajo grupal, reconocen fortalezas y debilidades individuales, valorando el trabajo colaborativo con pares que presentan habilidades complementarias, promoviendo la adquisición de este modelo en su práctica profesional. Si bien algunos estudios consideran otras didácticas como homólogos al $A B C$ (ej. aprendizaje basado en problemas), para términos de este estudio se considerará literatura que los diferencia en la preparación previa de la resolución de los casos, y el rol facilitador que cumple el académico guiando la discusión reflexiva (Srinivasan et al., 2007).

El objetivo principal de este estudio fue reportar el impacto de la incorporación de la didáctica educativa de $\mathrm{ABC}$ en una asignatura clínica curricular sobre el razonamiento clínico, pensamiento crítico, reflexión y habilidades comunicacionales en estudiantes de tercer año de kinesiología UC.

\section{Metodología}

Estudio descriptivo con análisis retrospectivo. Se utilizó la metodología de análisis de contenidos de la encuesta aplicada a los participantes, incluyendo comentarios relevantes luego de la aplicación de la didáctica educativa de ABC.

\section{Casos clínicos}

Los académicos participantes de este proyecto recibieron entrenamiento previo de expertos para la aplicación y evaluación de la didáctica de $A B C$, estableciendo en conjunto los instrumentos y objetivos de evaluación antes del comienzo de las actividades. Es así como se aplicó la misma pauta de evaluación para todos los grupos. Posteriormente, se diseñaron y desarrollaron un total de diez casos clínicos considerando los temas más comunes de la kinesiología cardiorrespiratoria, los que fueron aprobados con criterio de jueces por un panel de académicos expertos en el área. Cada académico guió el caso que desarrolló en virtud de su expertiz y experiencia clínica, de acuerdo a los objetivos y criterios de evaluación establecidos previamente con el equipo.

\section{Didáctica educativa}

La metodología docente del proyecto se incorporó en la asignatura de evaluación cardiorrespiratoria en kinesiología (KIN304a) impartida en el sexto semestre de la carrera de kinesiología UC, cuyo objetivo es que los estudiantes realicen adecuadamente el proceso de evaluación clínica con enfoque cardiorrespiratorio en pacientes reales, desarrollando un adecuado razonamiento 
clínico que les permita ser capaces de formular un diagnóstico kinesiológico e identificar problemas que sustenten la resolución terapéutica a plantear en niveles superiores.

La didáctica se realizó mediante trabajo en grupos de 6 a 7 estudiantes, quienes prepararon los casos clínicos con 2 semanas previas a la presentación al resto del curso. Cada grupo fue guiado por un académico participante de la asignatura quien seleccionaba las lecturas y recursos adicionales para que los estudiantes prepararan adecuadamente la actividad y fueran capaces de identificar los hechos, percepciones y contexto clínico entregado, reflexionando sobre lo aprendido e información faltante, y trabajando colaborativamente con sus pares. Cada grupo presentó con originalidad y creatividad el caso al resto de sus compañeros a través de diferentes métodos (ej: video, simulación, concurso, presentación, etc).

El tutor asignado al grupo incentivó la discusión con el resto del curso, el cual discutía en grupos pequeños respecto a preguntas que mantenían una secuencia establecida respecto al caso clínico presentado. En primer lugar, se buscó que los estudiantes identificaran qué estaba pasando (situación y contexto); después, que desarrollaran preguntas analíticas en base al caso clínico (¿por qué ocurre?) y finalmente, preguntas relacionadas con el desarrollo y resolución del caso (¿qué se puede hacer?; ¿qué puede anticiparse que pase?). El facilitador mantuvo el orden y guió la discusión a un ritmo adecuado para que todos los estudiantes comprendieran la situación sin restringir su participación, realizando preguntas que promovían el pensamiento crítico de cada caso, explicitando los patrones de ideas que surgían y otorgando información a medida que fue necesario.

El tiempo empleado en la discusión de cada caso fue alrededor de 90 minutos, y una vez finalizado, el académico guía presentó al curso contenidos adicionales como complemento de lo surgido en el desarrollo de la actividad. Para finalizar, cada grupo de estudiantes revisó nuevamente el caso, y en un plazo no mayor a 7 días entregó un resumen escrito de las conclusiones más relevantes, señalando los aspectos más enriquecedores que obtuvieron de los comentarios propios y de sus compañeros.

\section{Evaluación de la didáctica}

Al finalizar la asignatura, se invitó a los estudiantes mediante correo electrónico y enlace web a responder un cuestionario relacionado al logro de los objetivos de aprendizaje de la asignatura consistentes en desarrollar el razonamiento clínico, pensamiento crítico, reflexión y habilidades comunicacionales. Así, se diseñó una encuesta de 10 preguntas, cuyas respuestas consistían en calificar en una escala de 1 a 7 las afirmaciones que se planteaban, siendo
1 totalmente en desacuerdo, 2 en desacuerdo, 3 parcialmente en desacuerdo, 4 ni de acuerdo ni en desacuerdo, 5 parcialmente de acuerdo, 6 de acuerdo y 7 totalmente de acuerdo. Las respuestas se almacenaron de forma anónima en formato digital en la plataforma SurveyMonkey ${ }^{\oplus}$. El plazo máximo de respuesta para el cuestionario fue de 30 días.

\section{Evaluación del rendimiento académico}

Las instancias evaluativas de la asignatura no han variado entre las cohortes 2014 en adelante, siendo 2 interrogaciones teórica/práctica, 1 interrogación clínica y 5 visitas clínicas. Desde la cohorte 2017 en adelante se incorporó una pauta de evalaución similar a Mini-CEX utilizado en otra carrera de la salud de la Pontificia Universidad Católica de Chile (medicina), lo que implicó que el rendimiento en visitas clínicas de las cohortes 2014 a 2016 no sean comparabales a las 2017 y 2018. De esta manera, para evaluar si la metodología $A B C$ impactó en los indicadores de rendimiento académico, se comparó cada una de las instancias citadas previamente de las diferentes cohortes sin metodología de ABC (2014 a 2017), respecto a la cohorte intervenida (2018). El software estadístico utilizado fue GraphPad-Prism (v8.0) (San Diego, California, EE.UU). Se consideró para diferencias significativas un valor $p<0,05$.

\section{Resultados}

Un total de 75 participantes realizaron esta didáctica educativa, de los cuales 54 (72,0\%) respondieron la encuesta enviada al finalizar la asignatura. Todas las preguntas con sus respectivas respuestas se pueden observar en las tablas 1 y 2 . Éstas se presentan como porcentaje del total y se acompañan de las frases más reiterativas de los estudiantes.

\section{Razonamiento clínico}

En la pregunta $n^{\circ} 1$, el 66,7\% de los estudiantes refirió estar completamente de acuerdo y un $25,9 \%$ de acuerdo con que la metodología de $\mathrm{ABC}$ contribuyó a fomentar su razonamiento clínico, destacando que "permite desarrollar un algoritmo adecuado para detectar problemas kinesiológicos"; "fue fundamental porque permitía aplicar los contenidos del curso a medida que eran impartidos durante el semestre" y "ayudó mucho a perfeccionar la capacidad de razonamiento clínico".

En la pregunta $n^{\circ} 2$ que afirmaba que la metodología de $A B C$ permitió integrar conocimientos de otras asignaturas, el 37,0\% declaró estar de acuerdo con dicha premisa, un $26,0 \%$ totalmente de acuerdo, $18,5 \%$ ni de acuerdo ni en desacuerdo, 14,8\% parcialmente de acuerdo y un 3,7\% parcialmente en desacuerdo. 
Respecto a la pregunta $n^{\circ} 6$, el 62,9\% declaró estar totalmente de acuerdo con que contar con un tutor guía facilitó su aprendizaje, refiriendo que "facilitaba aclarar dudas y guiaba en los aspectos más importantes del caso" y que "ayudó contar con una persona con experiencia que guiara y transmitiera también su propio conocimiento"; 14,8\% declaró estar parcialmente de acuerdo mencionando que "facilita el aprendizaje, pero a veces es escaso el tiempo para reunirse con el tutor y eso dificulta un poco el avance con el trabajo". En este mismo contexto, en la pregunta $n^{\circ} 7$ el 59,2\% de los estudiantes declaró estar totalmente de acuerdo con que el tutor asignado lo guió durante el desarrollo del caso.

Tabla 1: Resultados de la encuesta (parte 1).

\begin{tabular}{|c|c|c|c|}
\hline $\mathbf{N}^{\circ}$ & Pregunta & Respuesta & Porcentaje (\%) \\
\hline \multirow{7}{*}{1} & \multirow{7}{*}{ ¿La metodología implementada contribuyó a fomentar su razonamiento clínico? } & 1 & 0,0 \\
\hline & & 2 & 0,0 \\
\hline & & 3 & 0,0 \\
\hline & & 4 & 3,7 \\
\hline & & 5 & 3,7 \\
\hline & & 6 & 25,9 \\
\hline & & 7 & 66,7 \\
\hline \multirow{7}{*}{2} & \multirow{7}{*}{ ¿La metodología $A B C$ le permitió integrar conocimientos de otras asignaturas? } & 1 & 0,0 \\
\hline & & 2 & 0,0 \\
\hline & & 3 & 3,7 \\
\hline & & 4 & 18,5 \\
\hline & & 5 & 14,8 \\
\hline & & 6 & 37,0 \\
\hline & & 7 & 26,0 \\
\hline \multirow{7}{*}{3} & \multirow{7}{*}{ ¿Los temas discutidos contribuyeron en su preparación para el campo clínico? } & 1 & 0,0 \\
\hline & & 2 & 0,0 \\
\hline & & 3 & 0,0 \\
\hline & & 4 & 0,0 \\
\hline & & 5 & 3,7 \\
\hline & & 6 & 26,0 \\
\hline & & 7 & 70,3 \\
\hline \multirow{7}{*}{4} & \multirow{7}{*}{ ¿Los temas analizados en los diferentes escenarios se asemejan a lo visto en campo clínico? } & 1 & 0,0 \\
\hline & & 2 & 0,0 \\
\hline & & 3 & 3,7 \\
\hline & & 4 & 0,0 \\
\hline & & 5 & 14,8 \\
\hline & & 6 & 29,7 \\
\hline & & 7 & 51,8 \\
\hline \multirow{7}{*}{5} & \multirow{7}{*}{ ¿Trabajar en el caso asignado, favoreció el trabajo en equipo? } & 1 & 0,0 \\
\hline & & 2 & 0,0 \\
\hline & & 3 & 3,7 \\
\hline & & 4 & 7,5 \\
\hline & & 5 & 25,9 \\
\hline & & 6 & 25,9 \\
\hline & & 7 & 37,0 \\
\hline
\end{tabular}

1: totalmente en desacuerdo; 2: en desacuerdo; 3: parcialmente en desacuerdo; 4: ni de acuerdo ni en desacuerdo; 5: parcialmente de acuerdo; 6: de acuerdo; 7: totalmente de acuerdo. 


\section{Rendimiento académico}

Las notas de las diferentes instancias evaluativas de las cohortes 2014 a 2017 fueron evaluadas mediante el test de Kolmogorov-Smirnov para determinar su distribución. En vista de la normalidad de los datos, se comparó el promedio de cada instancia de cada una de las cohortes 2014 a 2017 respecto a 2018, a través del test $t$-Student. Respecto a las interrogaciones teórica-práctica $(n=2)$, no hubo diferencias en las cohortes 2014 ( $p=0,078)$ y 2015 ( $p=0,069)$; mientras que fue mas baja en las cohortes 2016 ( $p=0.035)$ y 2017 $(p=0,034)$. Para la interrogación clínica con pacientes reales $(n=1)$ que se realiza próximo a la finalización de la asignatura, no hubo diferencias con la cohorte $2014(p=0,138)$, pero con las cohortes 2015 ( $p=0,045), 2016(p=0,028)$, y $2017(p=0,032)$ la cohorte 2018 obtuvo mejor calificación. Respecto al rendimiento de las visitas clínicas, sólo se compararon las cohortes que han utilizado el mismo instrumento de evaluación, así el rendimiento fue mas bajo en 2017 $(p=0,031)$. Por último, respecto al promedio final de la asignatura no hubo diferencias con 2014 ( $p=0.980$ ) y 2015 ( $p=0.786$ ), mientras que fue mas bajo en las cohortes $2016(p=0,034)$ y 2017 ( $p=0.041)$. Los datos se muestran en la tabla 3 y figura 1.

Respecto a si la metodología educativa impartida contribuyó al desempeño durante el desarrollo de la asignatura (pregunta $n^{\circ} 8$ ), $51,8 \%$ declaró estar totalmente de acuerdo, $33,4 \%$ de acuerdo y $14,8 \%$ parcialmente de acuerdo. Al respecto, los estudiantes refieren que la metodología "permite practicary desarrollar el análisis de contextos fisiopatológicos y exámenes comunes a la kinesiterapia cardiorrespiratoria", "ayudó a ganar mayor experiencia y dominar mejor los contenidos de la asignatura"y "es una forma diferente de aprender, es aprender haciendo".

Tabla 2: Resultados de la encuesta (parte 2).

\begin{tabular}{|c|c|c|c|}
\hline $\mathbf{N}^{\circ}$ & Pregunta & Respuesta & Porcentaje (\%) \\
\hline \multirow{7}{*}{6} & \multirow{7}{*}{ ¿Contar con un tutor asignado facilitó su aprendizaje? } & 1 & 0,0 \\
\hline & & 2 & 3,7 \\
\hline & & 3 & 0,0 \\
\hline & & 4 & 11,1 \\
\hline & & 5 & 14,8 \\
\hline & & 6 & 7,5 \\
\hline & & 7 & 62,9 \\
\hline \multirow{7}{*}{7} & \multirow{7}{*}{ ¿El tutor asignado lo guió durante el desarrollo del caso? } & 1 & 3,7 \\
\hline & & 2 & 3,7 \\
\hline & & 3 & 3,7 \\
\hline & & 4 & 3,7 \\
\hline & & 5 & 3,7 \\
\hline & & 6 & 22,3 \\
\hline & & 7 & 59,2 \\
\hline \multirow{7}{*}{8} & \multirow{7}{*}{ ¿La metodología $\mathrm{ABC}$ contribuyó en su desempeño durante el desarrollo de la asignatura? } & 1 & 0,0 \\
\hline & & 2 & 0,0 \\
\hline & & 3 & 0,0 \\
\hline & & 4 & 0,0 \\
\hline & & 5 & 14,8 \\
\hline & & 6 & 33,4 \\
\hline & & 7 & 51,8 \\
\hline \multirow{7}{*}{9} & \multirow{7}{*}{ ¿La metodología desarrollada, fomentó sus habilidades comunicacionales? } & 1 & 0,0 \\
\hline & & 2 & 0,0 \\
\hline & & 3 & 3,7 \\
\hline & & 4 & 7,4 \\
\hline & & 5 & 18,5 \\
\hline & & 6 & 29,6 \\
\hline & & 7 & 40,8 \\
\hline \multirow{7}{*}{10} & \multirow{7}{*}{ ¿El tiempo destinado, desde que recibió el caso hasta su presentación fue adecuado? } & 1 & 3,7 \\
\hline & & 2 & 3,7 \\
\hline & & 3 & 11,1 \\
\hline & & 4 & 3,7 \\
\hline & & 5 & 14,8 \\
\hline & & 6 & 11,1 \\
\hline & & 7 & 51,9 \\
\hline
\end{tabular}

1: totalmente en desacuerdo; 2: en desacuerdo; 3: parcialmente en desacuerdo; 4: ni de acuerdo ni en desacuerdo; 5: parcialmente de acuerdo; 6: de acuerdo; 7: totalmente de acuerdo. 


\section{Preparación para visitas clínicas}

Respecto a las visitas clínicas, en la pregunta $n^{\circ} 3$ el 70,3\% declaró estar totalmente de acuerdo y el $26,0 \%$ de acuerdo con que los temas discutidos en la metodología impartida en la asignatura, contribuyeron en la preparación para actividades en campo clínico, declarando que "la discusión de los temas ayudaba mucho al razonamiento de los casos que nos tocaban en las visitas en campo clínico y en cómo abordar a los pacientes"; "eran casos cotidianos y muy atingentes a lo que frecuentemente ven los kinesiólogos en el campo clínico". Un 3,7\% declaró estar parcialmente de acuerdo, refiriendo que "en algunas visitas clínicas estuvimos con pacientes cuyas patologías eran extrañas y poco frecuentes, y no se asemejaban mucho a lo desarrollado en los $A B C$ ".

Al consultar si los temas analizados en los diferentes escenarios se asemejan a lo visto en campo clínico (pregunta $n^{\circ} 4$ ), el 51,8\% declaró estar totalmente de acuerdo, un $29,7 \%$ de acuerdo, $14,8 \%$ parcialmente de acuerdo y $3,7 \%$ parcialmente en desacuerdo. Dentro de los comentarios más reiterativos al respecto se encuentra que "Ios temas analizados corresponden a las patologías más frecuentes"; "revisar temas que luego se transforman en patologías comunes en las visitas clínicas fomenta la seguridad para abordar al paciente"; "Ios $A B C$ son tan completos, que en las visitas clínicas cuesta menos el razonamiento clínico".

\section{Habilidades comunicacionales}

Respecto al trabajo en equipo y la comunicación entre pares, en la pregunta ${ }^{\circ} 5$ el $37,0 \%$ declaró estar totalmente de acuerdo con que trabajar en el caso asignado favoreció el trabajo en equipo, un 25,9\% declaró estar de acuerdo y otro $25,9 \%$ parcialmente de acuerdo con dicha premisa, destacando que "de todas maneras el trabajo en equipo se favorece, pero al ser varios integrantes, algunos trabajan más y otros menos"; "algunos compañeros son más empeñosos que otros, pero el trabajo en equipo siempre facilita la aparición de un integrante que toma el rol de líder y distribuye de forma equitativa las tareas, lo cual siempre es positivo"; "cada integrante del grupo aporta de forma diferente, lo cual enriquece sin duda el trabajo y fortalece el resultado final".

En la pregunta $n^{\circ} 9$, la cual afirmaba que la metodología desarrollada fomentó las habilidades comunicacionales, el 40,8\% respondió estar totalmente de acuerdo, 29,6\% de acuerdo, 18,5\% parcialmente de acuerdo, 7,4\% ni de acuerdo ni en desacuerdo y un 3,7\% de los encuestados respondió estar parcialmente en desacuerdo con dicha premisa. Al respecto, mencionan que "cuando uno como estudiante es el protagonista y en estricto rigor es quién enseña a los compañeros, se fomenta el desarrollo de habilidades comunicacionales y actitudinales. Uno se empodera y toma mayor seguridad".

\section{Discusión}

La incorporación de la didáctica de aprendizaje basado en casos $(A B C)$ en la asignatura de evaluación cardiorrespiratoria en kinesiología, favoreció el desarrollo de razonamiento clínico, pensamiento crítico, reflexión y habilidades comunicacionales en estudiantes de tercer año de kinesiología UC contribuyendo al logro de objetivos de aprendizaje. La didáctica facilitó la identificación de fortalezas y habilidades profesionales para la atención directa de pacientes. El escenario actual de la relación asistencial-docente entre carreras de la salud y campos clínicos en Chile, ha limitado las alianzas con los diferentes campos clínicos, acotando el número de experiencias clínicas disponibles para estudiantes de pregrado, lo cual dificulta el logro de objetivos de aprendizaje de las asignaturas clínicas (Ministerio de Salud, 2017). En este contexto, el ABC ha demostrado ser una metodología educativa que aumenta y complementa el conocimiento existente a través de un enfoque colaborativo basado en el trabajo en equipo, fomentando el aprendizaje al proporcionar integración vertical y horizontal del programa de estudios, contribuyendo así al logro de objetivos de aprendizaje, tal como lo evidencia nuestro estudio (Shigli et al., 2017).

En base al "Modelo de evaluación de programas de formación de Kirkpatrick", el cual es considerado el marco de referencia para determinar el impacto de programas de formación (Kirkpatrick, 1979; Kirkpatrick \& Kirkpatrick, 2012) el presente estudio muestra satisfacción con la didáctica educativa y la experiencia de aprendizaje por parte de los estudiantes (nivel 1: reacción), lo cual es relevante considerando que el clima de aprendizaje es fundamental para la adquisión del conocimiento (Ríos et al., 2010). En lo referente a la asimilación del contenido de la experiencia de aprendizaje (nivel 2: aprendizaje), este estudio muestra mejoras en los promedios obtenidos en las interrogaciones teóricas respecto a cohortes anteriores (ver tabla 3 y figura 1a), siendo indicadores a corto plazo de integración de contenido propio de la asignatura.

Con relación a la aplicación y transferencia del aprendizaje a situaciones reales (nivel 3: efectividad), los estudiantes perciben una mejor preparación para las actividades de campo clínico. Al respecto, existe una mejora en los indicadores de desempeño en situaciones clínicas reales, donde los promedios obtenidos en la interrogación práctica con pacientes reales (interrogación clínica) y en las visitas clínicas fueron mejores a los de cohortes previas que no realizaron la metodología $A B C$ (ver tabla 3 y figuras 1b y 1c). Estas evaluaciones son realizadas con pautas de evaluación creadas por comité de expertos de la carrera de kinesiología UC, que valoran los aspectos de razonamiento y análisis en un contexto clínico. Son aplicadas por académicos que han participado desde la primera 
versión en la asignatura (año 2014) y que están en conocimiento de los objetivos y la metodología de la didáctica de ABC. Así, se advierten mejoras en habilidades de análisis y resolución de problemas y mayores capacidades de razonamiento clínico, similar a lo reportado previamente en postgrado (Minghong et al., 2019). Si bien estas mejoras no pueden ser atribuidas exclusivamente a la didáctica de $A B C$, pues las características personales de los participantes, experiencias clínicas y otras actividades realizadas dentro de la asignatura pudieron también contribuir a los resultados; cohortes previas fueron evaluadas por los mismos académicos e instrumentos de evaluación, presentaban los mismos objetivos de aprendizaje, e incluso presentaron mayor cantidad de experiencias clínicas que los participantes de este estudio, lo que evidencia que el $A B C$ impactó positivamente en la aplicación y transferencia del aprendizaje, lo que se refleja en el mejor promedio final de la asignatura (figura 1d). Este resultado podría ser replicable en otras carreras del área de la salud que desarrollen actividades prácticas con pacientes reales, en las cuales el estudiante deba realizar un adecuado razonamiento clínico para identificar problemas y formular un diagnóstico.

Tabla 3: Comparación del promedio de calificaciones de las diferentes evaluaciones de la asignatura entre los estudiantes participantes del estudio y cohortes previas.

\begin{tabular}{l|c|c|c|c|c|c}
\hline \multicolumn{1}{c|}{ Indicador } & $\begin{array}{c}\mathbf{2 0 1 4} \\
(\mathbf{n = 5 4 )}\end{array}$ & $\begin{array}{c}\mathbf{2 0 1 5} \\
(\mathbf{n = 8 4 )}\end{array}$ & $\begin{array}{c}\mathbf{2 0 1 6} \\
(\mathbf{n = 7 8})\end{array}$ & $\begin{array}{c}\mathbf{2 0 1 7} \\
(\mathbf{n = 8 1 )}\end{array}$ & $\begin{array}{c}\text { Promedio } \\
\mathbf{2 0 1 4 - 2 0 1 7}\end{array}$ & $\begin{array}{c}\mathbf{2 0 1 8} \\
(\mathbf{n}=\mathbf{7 5})\end{array}$ \\
\hline Interrogaciones teóricas/prácticas (n=2) & 5,2 & 5,1 & 5,0 & 5,0 & 5,1 & 5,3 \\
\hline Interrogación clínica con pacientes reales & 5,7 & 5,6 & 5,1 & 5,3 & 5,4 & 5,9 \\
\hline $\begin{array}{l}\text { Visitas clínicas (n=5) } \\
\text { (mismo instrumento de evaluación) }\end{array}$ & - & - & - & 6,0 & 6,0 & 6,3 \\
\hline Promedio asignatura & 5,8 & 5,9 & 5,3 & 5,5 & 5,6 & 5,8 \\
\hline Reprobación de la asignatura $(\mathrm{n})$ & - & 1 & 1 & 1 & 1 & 0 \\
\hline
\end{tabular}

a)

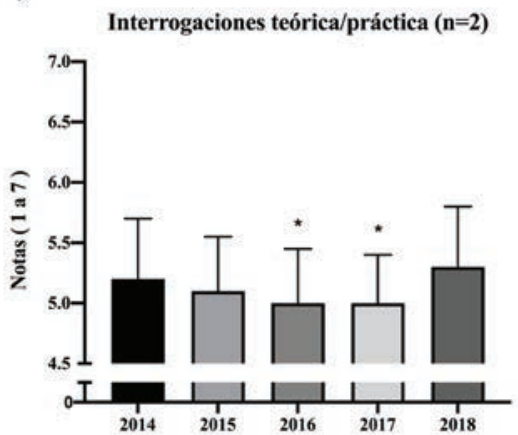

c)

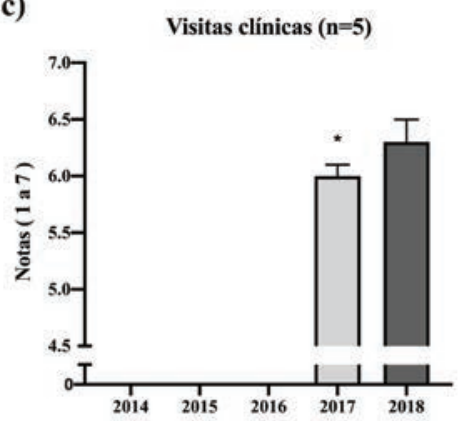

b)

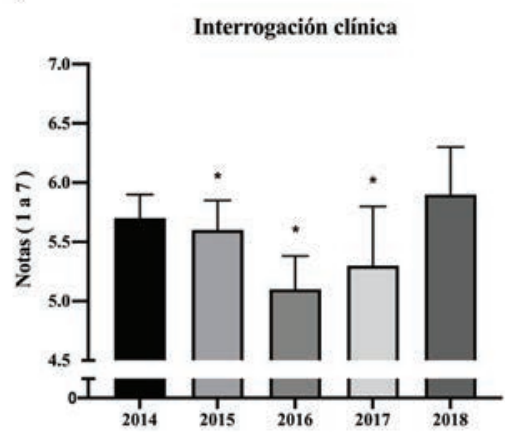

d)

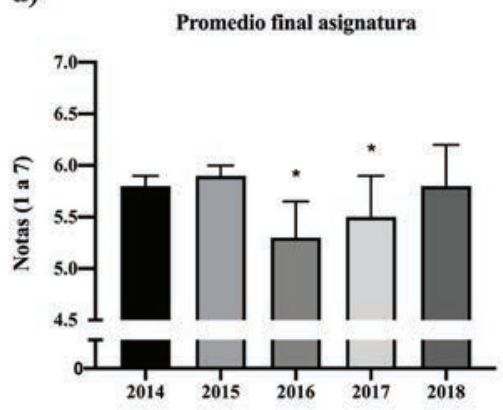

Figura 1: Comparación de calificaciones entre cohortes sin metodología $A B C$ respecto a cohorte con $A B C$. ${ }^{*} p<0,05$ respecto a cohorte 2018 (test $t$-Student). 
Finalmente, respecto a la influencia de la metodología en la organización y estructura de la asignatura y el plan de estudios (nivel 4: impacto), se evidencia una reducción del porcentaje de reprobación de la asignatura en los estudiantes que desarrollaron la metodología de $A B C$ (tabla 3 ). Sin embargo, se requiere más tiempo para establecer el impacto de este programa a largo plazo, donde se espera mejorar curvas de aprendizaje de habilidades clínicas del área cardiorrespiratorio, lo cual podría verse reflejado en el rendimiento académico en asignaturas de niveles superiores e internado profesional.

Respecto a la comunicación, este estudio evidencia que la didáctica educativa de $A B C$ fomentó el desarrollo de habilidades comunicacionales, pues los estudiantes manifiestan mayor confianza al momento de enfrentarse a sus pares y reconocen el trabajo en equipo como una instancia en que cada integrante tiene algo diferente que aportar. Esto es consistente con datos reportados donde la metodología $A B C$ demuestra ser una didáctica que incrementa la seguridad y satisfacción en el estudiante, junto con otorgar herramientas de comunicación y aumentar las oportunidades de interacción entre pares (Chowaniec et al., 2018).

En resumen, este estudio demostró que la incorporación de la didáctica educativa de aprendizaje basado en casos (ABC) en la asignatura de evaluación cardiorrespiratoria en kinesiología, favoreció el razonamiento clínico, reflexión y pensamiento crítico en estudiantes de kinesiología. De manera complementaria y como producto final del presente estudio, se confeccionó un libro digital que integra los diez diferentes escenarios clínicos desarrollados en la didáctica $A B C$. Cada caso es redactado como una historia que ejemplifica un paciente ficticio, enfatizando hallazgos clínicos y relacionándolo con contenidos teóricos; finalizando cada uno con preguntas abiertas que promueven la reflexión y el razonamiento clínico. Este material titulado: "Identificando problemas kinesiológicos: aprendizaje basado en casos" se encuentra incluido en la página web de la carrera de kinesiología y se encuentra disponible para libre descarga, constituyendo de esta forma un liderazgo en educación de pregrado en el área de la kinesiología cardiorrespiratoria, ampliando la visibilidad de la carrera más allá de las fronteras de la Universidad (Pontificia Universidad Católica de Chile, 2019).

\section{Agradecimientos}

Este estudio recibió apoyo financiero de Fondo de Desarrollo de la Docencia de la Pontificia Universidad Católica de Chile (FONDEDOC; Inv. Resp: FC).

\section{Conflictos de interés}

Los autores no reportan conflicto de interés.

\section{Contribuciones}

FCB: diseño de estudio, confección de casos clínicos, análisis de datos, redacción de manuscrito, aprobación final; YV: diseño de estudio, confección de casos clínicos, análisis de datos, aprobación final; MEsp: confección de casos clínicos, redacción de manuscrito, aprobación final; GV: confección de casos clínicos, aprobación final; GM: confección de casos clínicos, aprobación final; $\mathbf{M C h}$ : confección de casos clínicos, aprobación final; NN: confección de casos clínicos, aprobación final; ME: confección de casos clínicos, aprobación final; PG: confección de casos clínicos, aprobación final; TF: confección de casos clínicos, aprobación final; CRM: confección de casos clínicos, análisis de datos, redacción de manuscrito, aprobación final.

\section{Referencias}

Chowaniec J A, Doubleday A F, LeHew CW, Salzmann L B \& Koerber A. (2018). Timing of case-based discussions and educational outcomes for dental students. Journal of Dental Education 82, 510-514.

Epstein R M \& Hundert E M. (2002). Defining and assessing professional competence. Journal of the American Medical Association 287, 226-235.

Issenberg S B \& Scalese R J. (2018). Simulation in Health Care Education. Perspectives in Biology and Medicine 51, 31-46.

Kirkpatrick D. (1979). Techniques for Evaluating Training Programs. Training And Development Journal, 78-92.

Kirkpatrick D \& Kirkpatrick J. (2012). Evaluating training programs. Third edition. Berrett-Koehler. San Francisco.

Minghong B, Zhibiao Z, Jingru Y \& Wang Y. (2019). Comparison of case-based learning and traditional method in teaching postgraduate students of medical oncology. Medical Teacher 4, 1124-1128.

Ministerio de Salud; Subsecretaría de Salud Pública. (2012). LEY NúM. 20.584; Regula los derechos y deberes que tienen las personas en relación con acciones vinculadas a atención en salud. Accedido en: https://www.leychile.cl/Navegar?idNorma=1039348\&idVersion=2012-10-01 el 10 de Junio de 2019.

Pontificia Universidad Católica de Chile. (2019). Kinesiología: material académico de pregrado. Santiago, Chile. Accedido en: http://kinesiologia.uc.cl/material-academico/pregrado/ el 4 de Enero de 2020. 


\section{Contreras et al.}

Ríos D, Bozzo N, Marchant J \& Fernández P. (2010). Factores que inciden en el clima de aula universitario. Revista Latinoamericana de Estudios Educativos, 40, 105-126.

Shigli K, Aswini Y B, Fulari D, Sankeshwari B, Huddar D \& Vikneshan M. (2017). Case-based learning: A study to ascertain the effectiveness in enhancing the knowledge among interns of an Indian dental institute. Journal of Indian Prosthodontist Society 17, 29-34.
Srinivasan M, Wilkes M, Stevenson F, Nguyen T \& Slavin S. (2007). Comparing problem-based learning with case-based learning: Effects of a major curricular shift at two institutions. Academic Medicine 82, 74-82.

Williams B. (2005). Case based learning - a review of the literature: is there scope for this educational paradigm in prehospital education? Emergency Medicine Journal 22, 577-581. 